\title{
Detrimental Effects in Brillouin Distributed Sensors Caused By EDFA Transient
}

\author{
Cheng Feng*, ${ }^{*}$, Haritz Iribas², Jon Mariñelarena², Thomas Schneider $^{1}$, Alayn Loayssa ${ }^{2}$ \\ ${ }^{1}$ Institut für Hochfrequenztechnik, Technische Universität Braunschweig, 38106 Braunschweig, Germany \\ ${ }^{2}$ Departamento de Ingeniería Eléctrica y Electrónica, Universidad Pública de Navarra, 31006 Pamplona, Spain \\ *Email:cheng.feng@ihf.tu-bs.de
}

\begin{abstract}
We investigate the deleterious effect and the error in Brillouin optical time-domain analyzers induced by the combination of a low extinction ratio pulse generation with the transient behavior of erbium-doped fiber amplifiers.
\end{abstract}

OCIS codes: (060.2370) Fiber optics sensors, (290.5900) Scattering, stimulated Brillouin,

\section{Introduction and fundamentals}

The increasing demand for sensing, mainly in structural health monitoring, has raised wide investigations on Brillouin Optical Time Domain Analysis (BOTDA) in the last decades. The aim of these researches is commonly to either reduce the cost of the sensor or to improve its capability. One option to reduce the cost of the BOTDA system and also to generate fast optical pulses, is to employ an electro-optic modulator (EOM) to generate the pump wave. However, EOMs usually have a low extinction ratio (ER) and practical limitations such as measurement errors induced by ER of the pump pulse signal [1] are still limiting the performance of the sensor.

In this paper, we present an unknown distortion and a measurement error in BOTDA sensors due to the variation of the leakage power of the pump pulse when an erbium doped fiber amplifier (EDFA) is used to amplify a low ER pulse. Every EDFA has their own transient behavior when amplifying a pulse signal [2], resulting in a variation of the leakage power level before and after the pulse, as it is highlighted in Fig. 1(a). Moreover, as it has been studied, the probe wave experiences a gain due to a counter-propagated pump leakage [1]. Thus, the amplification given to the probe changes because of the different leakage power, so that the BOTDA trace will undergo a different amplification along the fiber, as it is illustrated in Fig. 1(b). Therefore, these variations introduce errors similar to the well-known non-local effects [3], since both are directly related to the Brillouin frequency shift (BFS) of the fiber.
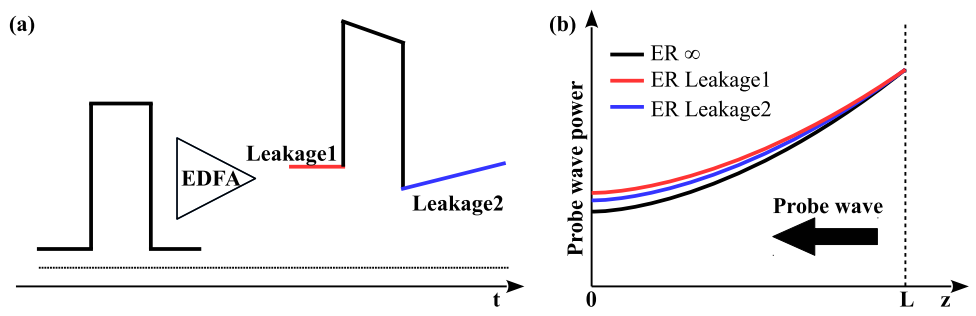

Fig. 1. (a) EDFA transient induced leakage distortion (b) leakage induced probe amplification.

\section{Experimental results}

We assembled a conventional dual-probe BOTDA setup [4], depicted in Fig. 2. In order to generate 60-ns pulses with different ERs, a Mach-Zehnder electro-optic modulator (MZM) and alternatively a semiconductor optical amplifier (SOA) were employed, obtaining a pulse ER of $23 \mathrm{~dB}$ and $45 \mathrm{~dB}$, respectively. The provided pump peak power and probe power were $18 \mathrm{dBm}$ and $-18 \mathrm{dBm}$, respectively, in order to avoid modulation instability and pulse depletion (checked in PD2). Two different EDFAs were tested to amplify the pump pulses and the transient behavior of the amplifier over the BOTDA trace is demonstrated. Finally, a $25 \mathrm{~km}$ standard single-mode fiber spool with a fairly uniform BFS distribution was deployed as sensing fiber applying 8000 averages. 


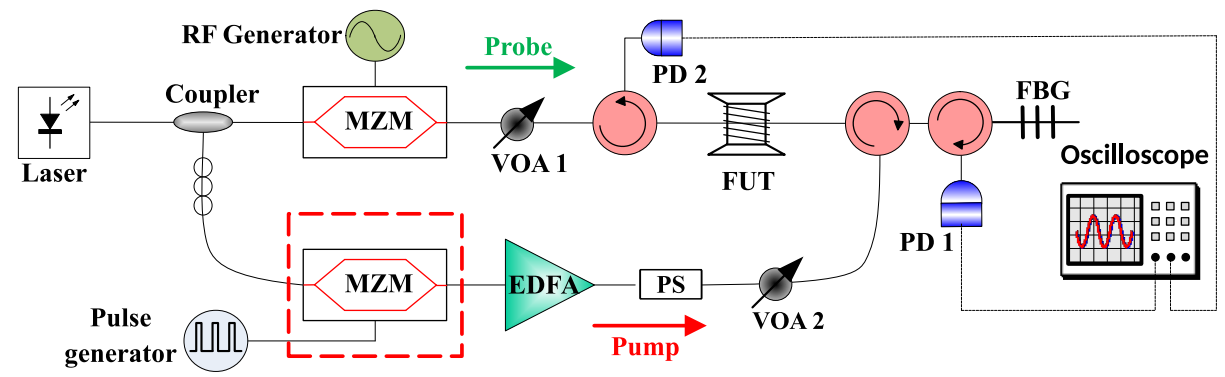

Fig. 2. Experimental setup. MZM: Mach-Zehnder modulator, VOA: Variable optical attenuator, PD: Photodiode, PS: Polarization scrambler, FBG: Fiber Bragg grating, FUT: Fiber under test.

Fig. 3(a) depicts the BOTDA traces at maximum BFS of the fiber for different ERs and EDFAs. Despite the same probe and pump power, a trace distortion for an ER of $23 \mathrm{~dB}$ is clearly visible while for $45 \mathrm{~dB}$ it is not. Since the SOA trace suffers no distortion, we regard the corresponding BFS measurement as reference for error comparison. The trace depletions with low ER are also different depending on the EDFA employed. Fig.3(b) shows the BFS measurement of the hot spot at the end of the fiber. Measurement error of $1.5 \mathrm{MHz}$ with EDFA 2 shows a good agreement with the larger trace distortion for EDFA 2 to EDFA 1 at the far end of the fiber.
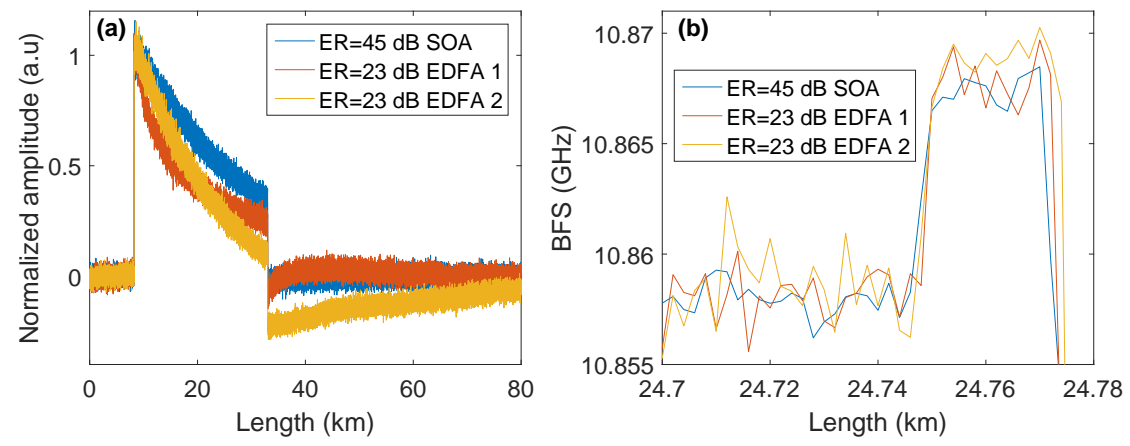

Fig. 3. (a) Normalized BOTDA traces and (b) BFS measurement of the hot spot.

\section{Conclusion}

In this paper, we have investigated for the first time to our knowledge an unknown distortion due to a combination of the finite ER and EDFA transient to the BOTDA measurement. Trace distortion and error in measuring the hot spot is experimentally performed. Measurement error should be expected in case an EDFA is used for amplification of the pump pulse with a finite ER in a BOTDA setup.

The authors wish to acknowledge the financial support of the German Research Foundation (DFG SCHN 716/13-1), Spanish Ministerio de Economía y Competitividad (TEC2016-76021-C2-1-R, TEC2013-47264-C2-2-R) and FEDER.

\section{References}

1. A. Zornoza, D. Olier, M. Sagues, and A. Loayssa, "Brillouin distributed sensor using RF shaping of pump pulses," Measurement Science and Technology 21, 94,021 (2010).

2. Y. Sun, J. L. Zyskind, and A. K. Srivastava, "Average inversion level, modeling, and physics of erbium-doped fiber amplifiers," IEEE Journal on Selected Topics in Quantum Electronics 3, 991-1007 (1997).

3. L. Thévenaz, S. F. Mafang, and J. Lin, "Effect of pulse depletion in a Brillouin optical time-domain analysis system," Optics Express 21, 14,017-14,035 (2013).

4. A. Minardo, R. Bernini, and L. Zeni, "A simple technique for reducing pump depletion in long-range distributed brillouin fiber sensors,” IEEE Sensors Journal 9, 633-634 (2009). 